\title{
Uplink Macro Diversity with Limited Backhaul Capacity
}

\author{
Amichai Sanderovich*, Oren Somekh ${ }^{\dagger}$, and Shlomo Shamai (Shitz)* \\ * Department of Electrical Engineering, Technion, Haifa 32000, Israel \\ $\dagger$ Department of Electrical Engineering, Princeton University, Princeton, NJ 08544, USA \\ Email: amichi@tx.technion.ac.il, orens@princeton.edu, sshlomo@ee.technion.ac.il
}

\begin{abstract}
In this contribution we present new achievable rates, for the non-fading uplink channel of a cellular network, with joint cell-site processing, where unlike previous results, the error-free backhaul network has finite capacity per-cell. Namely, the cell-sites are linked to the central joint processor via lossless links with finite capacity. The cellular network is modeled by the circular Wyner model, which yields closed form expressions for the achievable rates. For this idealistic model, we present achievable rates for cell-sites that use compress-and forward scheme, combined with local decoding, and inter-cell time-sharing. These rates are then demonstrated to be rather close to the optimal unlimited backhaul joint processing rates, already for modest backhaul capacities, supporting the potential gain offered by the joint cell-site processing approach.
\end{abstract}

\section{INTRODUCTION}

The growing demand for ubiquitous access to high-data rate services, has produced a huge amount of research analyzing the performance of wireless communications systems. Cellular systems are of major interest as the most common method for providing continuous services to mobile users, in both indoor and outdoor environments. In particular, the use of joint multicell processing (MCP) has been identified as a key tool for enhancing system performance (see [1] and references therein for recent results on multi-cell processing).

Since its introduction in [2], the Wyner cellular model has provided a framework for many works dealing with multicell processing. Albeit its simplicity, this model captures the essential structure of a cellular system and facilitates analytical treatment. The uplink channel of the Wyner linear and planar models are analyzed in [2] for optimal and linear minimum mean square error (MMSE) MCP receivers, and Gaussian channels. In [3], the Wyner model is extended to include fading channels and the performance of single and two cellsite processing under various setups is addressed. In [4] the results of [2] are extended to include fading channels.

All the above cited studies assume that the backhaul network connecting the cell-sites to the remote central processor (RCP) is error-free with infinite capacity. In this work we relax this assumption and allow each cell-site to connect to the RCP via a reliable error-free connection, but with limited capacity. Such a model suits cellular networks, where joint decoding can improve the overall network performance, with the underlying assumption that the received signals are forwarded to one location to be jointly processed. Since network resources are finite, in particular when the cell-sites are in fact "hot spots" with limited complexity, the inclusion of finite backhaul resources facilitates better prediction of the ultimate theoretical performance of MCP.

Recently, the common problem of nomadic terminals sending information to a remote destination via agents with lossless connections has been investigated in [5] and extended in [6] to include multiple-input multiple-output (MIMO) channels. These works focus on the nomadic regime, where the nomadic terminals use codebooks which are unknown to the agents, but fully known to the remote destination. Such setting suits the uplink channel of the limited backhaul cellular system with MCP, where the oblivious cell-sites play the role of the agents.

Using the techniques developed in [5][6], we assess the impact of limited backhaul capacity on the performance of various transmission schemes. Throughout this work we use a circular variant of the linear Wyner cellular setup [2], which provides an homogenous framework with respect to the mobile users and cell-sites. In particular we are interested in the asymptotic scenario of infinitely many nodes, where the circular and linear Wyner models are equivalent.

The rest of the paper is organized as follow. In Section II we define the system model. In Section III we prove an achievable rate for oblivious cell-sites, while in Section IV we consider the case where cell-sites perform partial local decoding and practice time-sharing. Numerical examples are presented in Section V, which demonstrate the effect of limited backhaul capacity on MCP performance. Several proofs are relegated to the Appendix.

\section{SYSTEM MODEL}

We consider the circular variant of the Wyner model [2], which includes an array of $N$ cell-sites, indexed by $j=$ $0, \ldots, N-1$, arranged on a circle. Each cell contains a single user ( $j$ user for the $j^{\text {th }}$ cell), which transmits $X_{j}$ to the channel, incorporating intra-cell time division multiple access (TDMA) transmission scheme. Each cell-site receives the transmission of the single user with independent interferences from the users of the adjacent cells and additive white circularly-symmetric complex Gaussian noise. The received signal at the $j^{\text {th }}$ cell-site for an arbitrary time index reads

$$
Y_{j}=X_{j}+\alpha\left(X_{[j-1]_{N}}+X_{[j+1]_{N}}\right)+Z_{j},
$$

where $[j]_{N} \triangleq j \bmod N$, and the inter-cell interference factor is $0 \leq \alpha \leq 1$. The additive noise $Z_{j}$ is circularly-symmetric 
complex Gaussian, with $\mathrm{E}\left|Z_{j}\right|^{2}=1$ and the transmission power is $\mathrm{E}\left|X_{j}\right|^{2}=P$, where the users use circularlysymmetric complex Gaussian codebooks. Using vector representation, expression (1) can be rewritten as

$$
Y_{\mathcal{N}}=H X_{\mathcal{N}}+Z_{\mathcal{N}}
$$

where $\mathcal{N}=\{0, \ldots, N-1\}$, and $Y_{\mathcal{N}}=\left\{Y_{0}, \ldots, Y_{N-1}\right\}$. The matrix $H$ is the $N \times N$ circulant channel transfer matrix, with first line $(1, \alpha, 0, \ldots, 0, \alpha)$. Each cell-site is connected to the RCP through a lossless link, with bandwidth of $C_{j}$ bits per channel use. The RCP jointly processes the signals and decodes the messages sent by all the users of the cellular system, where the code rate in bits per channel use of the $j^{\text {th }}$ user is $R_{j}$.

Using similar argumentation as in [2], it is easy to verify that intra-cell TDMA protocol is optimal in terms of the achievable throughput, for the non-fading homogenous setup considered.

\section{Oblivious Cell-Sites}

In this section we consider cell-sites which are oblivious to the users' codebooks and can not perform local decoding. Instead, each cell-site forwards a compressed version of $Y_{j}$, namely $U_{j}$, to the RCP, through the lossless link of bandwidth $C_{j}$. The RCP then receives the compressed $\left\{U_{j}\right\}$ and decodes the messages sent by all the users. We begin by stating the following achievable rate-region for the multi access channel (MAC).

Proposition 1: An achievable rate region for a general $N$ user MAC with oblivious $N$ cell-sites, connected by error-free limited capacities $\left\{C_{j}\right\}$ links to the $R C P$ is given by

$$
\begin{aligned}
& \forall \mathcal{L} \subseteq\{0, \ldots, N-1\}: \\
& \sum_{t \in \mathcal{L}} R_{t} \leq \min _{\mathcal{S} \subseteq \mathcal{N}}\left\{\sum_{j \in \mathcal{S}}\left[C_{j}-r_{j}\right]+I\left(X_{\mathcal{L}} ; U_{\mathcal{S}^{C}} \mid X_{\mathcal{L}^{C}}\right)\right\}
\end{aligned}
$$

where

$$
\begin{aligned}
P_{X_{\mathcal{N}}, U_{\mathcal{N}}, Y_{\mathcal{N}}}\left(X_{\mathcal{N}}, U_{\mathcal{N}}, Y_{\mathcal{N}}\right)=\prod_{j=1}^{N} P_{X_{j}}\left(X_{j}\right) \\
\prod_{j=1}^{N} P_{Y_{j} \mid X_{\mathcal{N}}}\left(Y_{j} \mid X_{\mathcal{N}}\right) \prod_{j=1}^{N} P_{U_{j} \mid Y_{j}}\left(U_{j} \mid Y_{j}\right),
\end{aligned}
$$

and $r_{j}=I\left(Y_{j} ; U_{j} \mid X_{\mathcal{N}}\right)$.

The proof outline appears in part A of the Appendix, is based on [5], and is given for $R_{j}=R$ (a single point on the achievable rate region boundaries) and equal capacities $C_{j}=C$, for the sake of brevity. The extension of the proof to the complete achievable rate region under any links' capacities is straightforward.

For the Gaussian channel, we use $\left\{X_{j}, U_{j}\right\}$ that are complex Gaussian, so that the joint probability (3) is Gaussian. It is noted that the Gaussian statistics is used due to the simplicity and relevance of the reported results, with no claim of optimality. For the Gaussian channel, the mutual information included in (2) boils down to [5],[6]

$$
\begin{aligned}
& I\left(X_{\mathcal{L}} ; U_{\mathcal{S}^{C}} \mid X_{\mathcal{L}^{C}}\right)= \\
& \quad \log _{2} \operatorname{det}\left(I+P \operatorname{diag}\left(1-2^{-r_{j}}\right)_{j \in \mathcal{S}^{C}} H_{\mathcal{S}^{C} \mathcal{L}} H_{\mathcal{S}^{C} \mathcal{L}}^{*}\right),
\end{aligned}
$$

where $H_{\mathcal{S}^{C} \mathcal{L}}$ is the transfer matrix between the output vector $Y_{\mathcal{S}^{C}}$ and the input vector $X_{\mathcal{L}}$, and $r_{j}$ are positive parameters that are subjected to optimization over $0 \leq r_{j} \leq C_{j}$. Focusing on the setup at hand, with $H_{i, j}$ which is zero for $N-1>$ $|i-j|>1$, equation (2) becomes

$\sum_{t \in \mathcal{L}} R_{t} \leq \min _{\mathcal{S} \subseteq[\mathcal{L}+1]_{N} \cup[\mathcal{L}-1]_{N} \cup \mathcal{L}} \sum_{j \in \mathcal{S}}\left[C_{j}-r_{j}\right]+I\left(X_{\mathcal{L}} ; U_{\mathcal{S}^{C}} \mid X_{\mathcal{L}^{C}}\right)$,

where $[\mathcal{L} \pm 1]_{N} \triangleq\{j: j=(i \pm 1) \bmod N, i \in \mathcal{L}\}$. Let us define $H_{\mathcal{S}}=H_{\mathcal{S N}}$, which is the transfer matrix between $X_{\mathcal{N}}$ and $Y_{\mathcal{S}}$.

Hereafter, we limit our attention to the symmetric case of $C_{i}=C$ for all cell-sites, and $R_{t}=R$ for all users. By symmetry and concavity, this limits the optimal $r_{j}$ to be invariant with respect to $j: r_{j}=r$, and the sum-rate inequality $(\mathcal{L}=\{0, \ldots, N-1\})$ to be the dominant inequality in (2). Consequently we get the following.

Corollary 1: An achievable rate for the circular Wyner model with equal capacity links $C$, equal rate users and oblivious cell-sites is given by

$$
\begin{aligned}
R_{o b l}=\frac{1}{N} \max _{0 \leq r}\left\{\min _{\mathcal{S} \subseteq \mathcal{N}}\{|\mathcal{S}|[C-r]\right. \\
\left.\left.\quad+\log _{2} \operatorname{det}\left(I+P\left(1-2^{-r}\right) H_{\mathcal{S}^{C}} H_{\mathcal{S}^{C}}^{*}\right)\right\}\right\} .
\end{aligned}
$$

This rate is achieved with complex Gaussian $\left\{U_{j}, X_{j}\right\}$.

Next, we need to calculate the logarithm of the determinant of (5). In the case where no inter-cell interference is presence $(\alpha=0)$, it is easily verified that $H_{\mathcal{S}^{C}} H_{\mathcal{S}^{C}}^{*}$ is an $\left|\mathcal{S}^{C}\right|$ identity matrix, and in this case the rate equals the rate achieved by an equivalent single-user single-agent channel [5], which is given by $\log _{2}\left(1+P \frac{2^{C}-1}{2^{C}+P}\right)$.

For $\alpha>0$, we focus on the case where the number of cells $N$ is large. An achievable rate for this asymptotic scenario is given by the following proposition, which is the main result of this work.

Proposition 2: An achievable rate for the circular Wyner model with equal limited capacities, oblivious cell-sites and infinite number of cells $(N \rightarrow \infty)$, is given by

$$
R_{o b l}=F\left(r^{*}\right)
$$

where $r^{*}$ is the solution of

$$
F\left(r^{*}\right)=C-r^{*}
$$

and

$$
F(r)=\int_{0}^{1} \log _{2}\left(1+P\left(1-2^{-r}\right)(1+2 \alpha \cos 2 \pi \theta)^{2}\right) d \theta \text {. }
$$


Notice that when $C \rightarrow \infty$, then also $r^{*} \rightarrow \infty$, and (6) boils down to the per-cell sum-rate capacity of the Wyner model with optimal joint processing and unlimited backhaul capacity [2]

$$
R_{o b l}=F(\infty)=\int_{0}^{1} \log _{2}\left(1+P(1+2 \alpha \cos 2 \pi \theta)^{2}\right) d \theta .
$$

For finite $C$, the implicit equation (7) is easily solved numerically, since $F(r)$ is monotonic. The following lemma, which is proved in part B of the Appendix, is required for the proof of Proposition 2.

Lemma 1: Any subset $\mathcal{S}$ such that $|\mathcal{S}|=f(N)$ $\left(f: \mathbb{R}_{+} \mapsto \mathbb{R}_{+}, \lim _{N \rightarrow \infty} \frac{f(N)}{N}=\lambda, 0 \leq \lambda \leq 1\right)$, which minimizes equation (5), when $N \rightarrow \infty$, includes only consecutive indices (considering also modulo operation). $\mathcal{S}^{(c)}$.

Denote a subset which contains only consecutive indices by

Proof of Proposition 2 (outline): First, note that applying Szegö's theorem [2], to $\log _{2} \operatorname{det}\left(I+P\left(1-2^{-r}\right) H_{\mathcal{S}^{(c)}} H_{\mathcal{S}^{(c)}}^{*}\right)$ when $\left|\mathcal{S}^{(c)}\right| \rightarrow \infty$, we get the following simple explicit expression

$\lim _{\left|\mathcal{S}^{(c)}\right| \rightarrow \infty} \frac{1}{\left|\mathcal{S}^{(c)}\right|} \log _{2} \operatorname{det}\left(I+P\left(1-2^{-r}\right) H_{\mathcal{S}^{(c)}} H_{\mathcal{S}^{(c)}}^{*}\right)=F(r)$.

Let us define $\left|\mathcal{S}^{(c)}\right|=s$, so that

$$
\log _{2} \operatorname{det}\left(I+P\left(1-2^{-r}\right) H_{\mathcal{S}^{(c)}} H_{\mathcal{S}^{(c)}}^{*}\right)=s F(r)+\epsilon(s),
$$

where $\lim _{s \rightarrow \infty} \epsilon(s) / s=0$.

Secondly, from Lemma 1 , when $N \rightarrow \infty$, the minimum in equation (5) is within the subspace of subsets that contain only consecutive indices $\left\{\mathcal{S}^{(c)}\right\}$. Combining (9), when $N \rightarrow \infty$, equation (5) becomes

$$
\begin{aligned}
R_{o b l}= & \lim _{N \rightarrow \infty}\left\{\operatorname { m a x } _ { 0 \leq r } \left\{\min _{0 \leq s \leq N}\right.\right. \\
& \left.\left.\left\{\frac{N-s}{N}[C-r]+\frac{s}{N} F(r)+\frac{\epsilon(s)}{N}\right\}\right\}\right\} \\
= & \max _{0 \leq r}\left\{\min _{0 \leq \lambda \leq 1}\{(1-\lambda)[C-r]+\lambda F(r)\}\right\} .
\end{aligned}
$$

Since $F(r)$ is monotonic increasing, (10) is maximized by $r^{*}$, which is defined by $F\left(r^{*}\right)=C-r^{*}$.

\section{CEll-Sites With DeCODing}

In order to better utilize the bandwidth between the cellsites and the RCP, we may consider using local decoding at the cell-sites. In this case the cell-sites should be aware of the associated codebooks, and thus do not operate in the nomadic regime [5]. It is noted that in general, decoding decreases the noise uncertainty, thus increasing the efficiency of backhaul usage.

In this section we present an intuitive, simple scheme which provides an achievable rate. According to this scheme, each user employs rate splitting and divides its message into two parts: one that is decoded at the RCP and another that is decoded at the local cell-site. In this case the message which is intended for the RCP to decode, interferes with the decoding of the relevant message at the cell-site. Let the power used for the former be $\beta P$ and the latter $(1-\beta) P$, where $0 \leq \beta \leq 1$. There are two strategies for the cell-site to execute: to decode only its local user's message, or to decode also the interfering users' messages, emerging from the two neighboring cells (see [3] Section III.D). Such approach allows decoding of messages with rate of

$$
\begin{aligned}
& R_{d}=\max \left\{\log _{2}\left(1+\frac{(1-\beta) P}{1+\left(\beta+2 \alpha^{2}\right) P}\right),\right. \\
& \min \left\{\frac{1}{2} \log _{2}\left(1+\frac{(1-\beta) 2 \alpha^{2} P}{1+\beta\left(1+2 \alpha^{2}\right) P}\right),\right. \\
&\left.\left.\frac{1}{3} \log _{2}\left(1+\frac{\left(1+2 \alpha^{2}\right)(1-\beta) P}{1+\beta\left(1+2 \alpha^{2}\right) P}\right)\right\}\right\} .
\end{aligned}
$$

Forwarding the decoded information through the lossless links reduces the bandwidth available for the compression, so the achievable rate is $R_{s d}(C)$ (sd stands for separate decoding)

$$
R_{s d}(C)=\max _{\beta}\left\{F_{\beta}\left(r_{d}^{*}\right)+\hat{R}_{d}(\beta)\right\},
$$

where $\hat{R}_{d}(\beta)=\min \left\{R_{d}(\beta), C\right\}, r_{d}^{*}$ is the solution of

$$
F_{\beta}\left(r_{d}^{*}\right)=C-\hat{R}_{d}(\beta)-r_{d}^{*},
$$

and $F_{\beta}(r)$ is defined as $F(r)$ from (8) while replacing $P$ with $\beta P$

$$
F_{\beta}(r)=\int_{0}^{1} \log _{2}\left(1+\beta P\left(1-2^{-r}\right)(1+2 \alpha \cos 2 \pi \theta)^{2}\right) d \theta .
$$

For $\alpha=0$ this scheme is optimal, since there is no intercell interference and each cell-site can decode messages at the same rate as the RCP can.

Note that the rate $R_{s d}(C)$ is not concave in $C$ in general, thus time-sharing may improve the achievable rate, which leads to the following (ch stands for the convex-hull).

Proposition 3: An achievable rate of the rate-splitting scheme deployed in the infinite circular Wyner model with limited equal capacities $C$, is given by

$$
\begin{aligned}
& R_{s d c h, 1}= \\
& \max _{\lambda, C_{1}, C_{2}: \lambda C_{1}+(1-\lambda) C_{2} \leq C}\left\{\lambda R_{s d}\left(C_{1}\right)+(1-\lambda) R_{s d}\left(C_{2}\right)\right\} .
\end{aligned}
$$

In fact, numerical calculations reveal that a good strategy is to do time-sharing between the two approaches: using decoding at the cell-sites, with no decoding at the RCP, and doing decoding only at the RCP (10), rather than using the mixed approach of (12). Thus, defining $t=\hat{R}_{d}(0)$, the rate $R_{s d c h, 1}$ of (13) can be written as

$$
R_{d e c}(P, C)=\max _{r \geq r^{*}}\left\{t+(C-t) \frac{F(r)-t}{F(r)+r-t}\right\},
$$


and $r^{*}$ is calculated by (7). The derivation of (14) is based on time-sharing between the point $(t, t)$ and the concave curve $(F(r)+r, F(r))$. It is noted that the maximizing $r$ must be larger than $r^{*}$, thus limiting the optimization range.

It is expected that decoding at the cell-site will be beneficial when $\alpha$ is small (low inter-cell interference), or when $C$ is small, so that decoding before transmission saves on bandwidth, which otherwise would have been wasted on noise quantization.

\section{A. Adding Inter-Cell Time-Sharing}

Another improvement that can be made to the overall performance is by allocating a fraction of the transmission interval in which adjacent users are not active simultaneously. Therefore allowing the cell-sites to decode their messages without interference during this fraction of time (see [3] Section III.D). For the circular Wyner model with $N$ even, it means that odd and even cells are active alternately in time, hence the acronym inter-cell time-sharing (ICTS). In the following we restrict our attention to even $N$. Redivide the time frames, such that every user is active for $(1-\theta)$ of the time $\left(0 \leq \theta \leq \frac{1}{2}\right)$, indicating that the time fraction used for transmitting in the interference-free phase is $\theta$. The time used for the previous rate-splitting scheme is thus $1-2 \theta$. Since the users do not transmit all the time, they can scale their transmission power by a factor of $\frac{1}{1-\theta}$. The resulting rate is

$$
\begin{aligned}
& R_{I C T S}=\max _{0 \leq \theta \leq 0.5}\left\{R_{d, 2}+\right. \\
&\left.(1-2 \theta) R_{\text {dec }}\left(\frac{P}{1-\theta}, \frac{1}{1-2 \theta}\left(C-R_{d, 2}\right)\right)\right\},
\end{aligned}
$$

where $R_{d, 2}$ is the rate of the message sent in the interferencefree phase, which equals

$$
R_{d, 2}=\min \left\{\theta \log _{2}\left(1+\frac{P}{1-\theta}\right), C\right\} .
$$

Finally, using (14) we get the following.

Proposition 4: An achievable rate of the ICTS with cell-site decoding, deployed in the infinite circular Wyner model with limited equal capacities, is given by

$$
\begin{aligned}
& R_{I C T S}=\max _{0 \leq \theta \leq 0.5}\left\{R_{d, 2}+\right. \\
& \left.(1-2 \theta) \max _{r \geq r_{t s}^{*}}\left\{t+\left(\frac{C-R_{d, 2}}{1-2 \theta}-t\right) \frac{F(r)-t}{F(r)+r-t}\right\}\right\},
\end{aligned}
$$

where $t=\min \left\{C-R_{d, 2},\left.R_{d}\right|_{\beta=0, \frac{P}{1-\theta}}\right\}$ and $r_{t s}^{*}$ is calculated by

$$
\left.F_{\beta}\left(r_{t s}^{*}\right)\right|_{\beta=\frac{1}{1-\theta}}=C-R_{d, 2}-r_{t s}^{*} .
$$

\section{NUMERICAL RESUlts}

Achievable rates of the considered three schemes, are plotted in Figure 1 for SNR $P=10[\mathrm{~dB}]$, and several backhaul capacity values, as functions of the inter-cell interference factor $\alpha$. Examining the curves, it is observed that in order

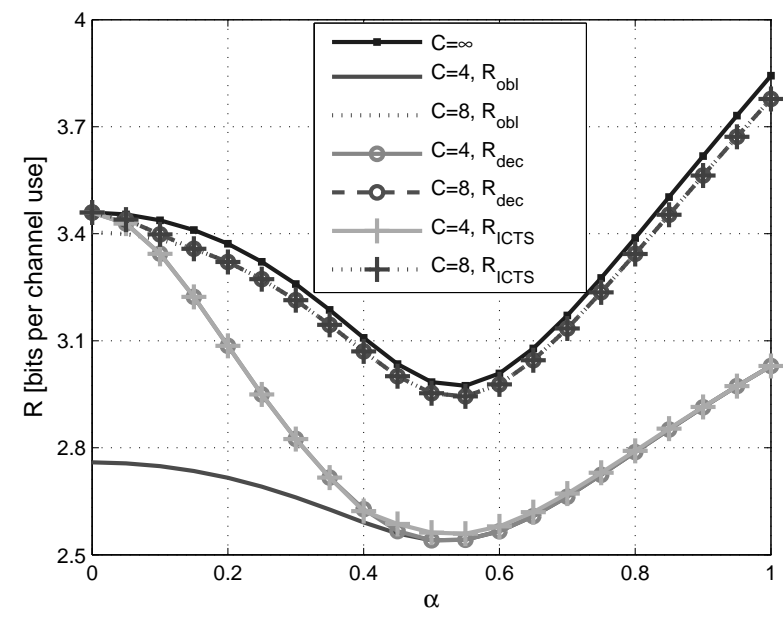

Fig. 1. The achievable rates $R_{o b l}, R_{d e c}$ and $R_{I C T S}$ are plotted as functions of the inter-cell interference $\alpha$ for $C=4,8, \infty$ and user SNR $P=10$ [dB].

to approach the rates achieved with $C=\infty$ across the whole region $0 \leq \alpha \leq 1$, we need an overhead with approximately twice as much bandwidth for the backhaul network $(C=2 R)$.

The gain of the cell-site decoding scheme is prominent when the inter-cell interference is low, but also noticeable even for rather high interference levels, when $C$ is low.

Interestingly, the ICTS scheme provides only a slight improvement over the cell-site decoding scheme. A possible explanation for this phenomenon is that for $\theta=\frac{1}{2}$ (no overlapping between transmissions of adjacent users), the achievable rate reads $R_{I C T S}=0.5 \log _{2}(1+2 P)$, which for $P=10$ roughly equals 2.2 [bits/channel use], which is significantly lower than all the considered schemes.

Another observation is that for high backhaul capacities, the rates of the three schemes approach the optimal performance. As expected for $\alpha=0$, the cell-site decoding and ICTS schemes rates are optimal independently of $C$, since no intercell interferences are present.

\section{CONCLUding REMARKS}

In this paper we considered the circular Wyner model, with limited backhaul capacity. Achievable rates were presented for the case of cell-sites which use signal processing alone, and also combined with decoding. Both considered no network planning, so inter-cell interferences dominate. Achievable rates were derived also for time-sharing based, optimized network planning. Numerical calculations reveal that unlimited optimal joint processing performance can be closely approached with rather limited backhaul capacity, and that network planning does not add significant performance gains.

The faded Wyner model with limited backhaul capacity is currently under study.

\section{APPENDIX}

\section{A. Proof Outline of Proposition 1}

Assuming transmission over $n$ channel uses, we generate the random codebooks for the users by $\left\{X_{j}\right\}$, and the random quantization codebooks (each of size $2^{n R}$ ) by $\left\{U_{j}\right\}$. Each 
quantization codebook is randomly partitioned into $2^{n C}$ bins, so that each bin consists of $2^{n[\hat{R}-C]}$ codewords.

Each cell-site uses the joint typicality criteria to compress the received channel output $Y_{j}^{n}$ into $U_{j}^{n}$, and then sends the resulting bin index (instead of the of the index of $U_{j}^{n}$, in order to save bandwidth) to the RCP through the lossless link. An error in this stage occurs with arbitrary low probability of error, as long as $\hat{R} \geq I\left(Y_{j} ; U_{j}\right)$.

The RCP receives the bins' indices, and looks for a jointly typical $\left\{X_{j}, U_{j}\right\}$, where the $\left\{U_{j}\right\}$ are taken from the bins indicated by the cell-sites.

The probability that no set $\left\{X_{j}, U_{j}\right\}_{j=1}^{N}$ is found to be jointly typical is arbitrary small with increasing $n$, due to the generalized Markov lemma [5]. The probability of a set with erroneous $X_{\mathcal{L}}$ and $U_{\mathcal{S}}$ (where $\mathcal{S}, \mathcal{L} \subseteq \mathcal{N}$ ) to be jointly typical is upper bounded by

$$
\begin{aligned}
P_{e} \leq 2^{n\left[|\mathcal{L}| R+|\mathcal{S}|(\hat{R}-C)+h\left(X_{\mathcal{N}}, U_{\mathcal{N}}\right)\right]} \\
\cdot 2^{-n\left[h\left(X_{\mathcal{L}^{C}}, U_{\mathcal{S}^{C}}\right)+h\left(X_{\mathcal{L}}\right)+\sum_{j \in \mathcal{S}} h\left(U_{j}\right)\right]} .
\end{aligned}
$$

Hence, the probability of error is arbitrary small as long as

$$
\begin{aligned}
&|\mathcal{L}| R \leq|\mathcal{S}|(C-I(U ; Y))- h\left(X_{\mathcal{L}}, U_{\mathcal{S}} \mid X_{\mathcal{L}^{C}}, U_{\mathcal{S}^{C}}\right) \\
&+|\mathcal{L}| h(X)+|\mathcal{S}| h(U),
\end{aligned}
$$

where the cell index $j$ is omitted due to symmetric argumentation.

Noting that $Y_{i}-X_{\mathcal{N}}-Y_{j}$ and $\left\{X_{\mathcal{N}}, U_{i}\right\}-Y_{j}-U_{j}$ are Markov chains for $i \neq j$, and that users cooperation is not allowed, it is easy to verify that the following equalities hold

$$
\begin{aligned}
h\left(U_{j} \mid Y_{j}\right) & =h\left(U_{j} \mid Y_{j}, X_{\mathcal{N}}\right) \\
h\left(X_{\mathcal{L}}\right) & =h\left(X_{\mathcal{L}} \mid X_{\mathcal{L}^{C}}\right) \\
h\left(X_{\mathcal{L}}, U_{\mathcal{S}} \mid X_{\mathcal{L}^{C}}, U_{\mathcal{S}^{C}}\right) & =h\left(X_{\mathcal{L}} \mid X_{\mathcal{L}^{C}}, U_{\mathcal{S}^{C}}\right)+|\mathcal{S}| h\left(U \mid X_{\mathcal{N}}\right) .
\end{aligned}
$$

Applying these to (17) yields

$$
\left.|\mathcal{L}| R \leq|\mathcal{S}|\left(C-I\left(U ; Y \mid X_{\mathcal{N}}\right)\right)+I\left(X_{\mathcal{L}} ; U_{\mathcal{S}^{C}} \mid X_{\mathcal{L}^{C}}\right)\right) .
$$

Noting that the last condition should hold for any $\mathcal{S}$ and $\mathcal{L}$ completes the proof.

\section{B. Proof of Lemma 1}

We prove that $\mathcal{S}$ which minimizes

$$
\lim _{N \rightarrow \infty} \frac{1}{N} I\left(X_{\mathcal{N}} ; U_{\mathcal{S}}\right)=\lim _{N \rightarrow \infty} \frac{1}{N} \log _{2} \operatorname{det}\left(I+P^{\prime} H_{\mathcal{S}} H_{\mathcal{S}}^{*}\right),
$$

when $|\mathcal{S}|=f(N),\left(f: \mathbb{R}_{+} \mapsto \mathbb{R}_{+}, \lim _{N \rightarrow \infty} \frac{f(N)}{N}=\lambda\right.$, $0 \leq \lambda \leq 1)$, is composed of only consecutive indices.

Following the method used in [8] to derive a lower bound on the capacity of the Gaussian erasure channel, the proof here uses an analogy between the multi-cell setup and an intersymbol interference (ISI) channel, combined with a recently reported relation between the MMSE and the mutual information [7].

Proof: Denote by $E_{i}$, the MMSE of estimating $X_{i}$ from $U_{\mathcal{N}}$. Further, denote by $E_{i}(\mathcal{S})$, the MMSE of estimating $X_{i}$ from $U_{\mathcal{S}}$. Naturally

$$
\forall \mathcal{S} \subseteq \mathcal{N}, i \in \mathcal{N}: E_{i}(\mathcal{S}) \geq E_{i}
$$

Next, we use the following relation between the MMSE and the mutual information [7], to write

$$
\frac{d}{d P} I\left(X_{\mathcal{N}} ; U_{\mathcal{S}}\right)=\sum_{i=0}^{N-1} E_{i}(\mathcal{S})
$$

From (18), we can write

$$
\sum_{i=0}^{N-1} E_{i}(\mathcal{S}) \geq \frac{f(N)}{N} \sum_{i=0}^{N-1} E_{i}
$$

Combining (19) and (20) yields

$$
I\left(X_{\mathcal{N}} ; U_{\mathcal{S}}\right) \geq \frac{f(N)}{N} \int_{0}^{P^{\prime}} \sum_{i=0}^{N-1} E_{i} d P=\frac{f(N)}{N} I\left(X_{\mathcal{N}} ; U_{\mathcal{N}}\right) .
$$

On the other hand, in the asymptotic regime, for consecutive indices set $\mathcal{S}^{(c)}$, where $\lim _{N \rightarrow \infty} \frac{\left|\mathcal{S}^{(c)}\right|}{N}=\lambda$

$$
\lim _{N \rightarrow \infty} \frac{1}{N} \sum_{i \in \mathcal{S}^{(c)}} E_{i}\left(\mathcal{S}^{(c)}\right)=\lambda \lim _{N \rightarrow \infty} \frac{1}{N} \sum_{i=0}^{N-1} E_{i} .
$$

This is because the equivalent ISI channel is stationary, and since the right hand side of (22) exists (attainable from applying Szegö's theorem to the mutual information and then differentiating with respect to $P^{\prime}$ ). By integrating both flanks of equation (22) we get that

$$
\lim _{N \rightarrow \infty} \frac{1}{N} I\left(X_{\mathcal{N}} ; U_{\mathcal{S}^{(c)}}\right)=\lambda \lim _{N \rightarrow \infty} \frac{1}{N} I\left(X_{\mathcal{N}} ; U_{\mathcal{N}}\right)
$$

Equation (23) together with (21) proves the lemma.

\section{ACKNOWLEDGMENT}

The research was supported by the REMON consortium for wireless communication and a Marie Curie Outgoing International Fellowship within the 6th European Community Programme.

\section{REFERENCES}

[1] S. Shamai, O. Somekh, and B. M. Zaidel, "Multi-cell communications: An information theoretic perspective," in Proceedings of the Joint Workshop on Communications and Coding (JWCC'04), Donnini, Florence, Italy, Oct. 2004.

[2] A. Wyner, "Shannon theoretic approach to a Gaussian cellular multiple access channel," IEEE Trans. Inform. Theory, vol. 40, no. 6, pp. 17131727, Nov 1994.

[3] S. Shamai and A. Wyner, "Information-theoretic considerations for symmetric cellular, multiple-access fading channels - part I," IEEE Trans. Inform. Theory, vol. 43, no. 6, pp. 1877-1894, Nov 1997.

[4] O. Somekh and S. Shamai, "Shannon-theoretic approach to a Gaussian cellular multi-access channel with fading," IEEE Trans. Inform. Theory, vol. 46, no. 4, pp. 1401-1425, July 2000.

[5] A. Sanderovich, S. Shamai, Y. Steinberg, and G. Kramer, "Communication via decentralized processing," Submitted to IEEE Trans. Inform. Theory.

[6] A. Sanderovich, S. Shamai, Y. Steinberg, and M. Peleg, "Decentralized receiver in a MIMO system," in Proc. of IEEE Int. Symp. Info. Theory (ISIT'06), Seattle, WA, July 2006, pp. 6-10.

[7] D. Guo, S. Shamai, and S. Verdú, "Mutual information and minimum mean-square error in Gaussian channels," IEEE Trans. Inform. Theory, vol. 51, no. 4, pp. 1261-1282, April 2005.

[8] A. M. Tulino, S. Verdú, G. Caire, and S. Shamai, "Capacity of the Gaussian erasure channel," Submitted to IEEE Trans. Inform. Theory. 